\title{
ORTHOMODULAR LATTICES CAN BE CONVERTED INTO LEFT RESIDUATED L-GROUPOIDS
}

\author{
IVAN CHAJDA AND HELMUT LÄNGER
}

Received 18 September, 2015

\begin{abstract}
We show that every orthomodular lattice can be considered as a left residuated 1groupoid satisfying divisibility, antitony, the double negation law and three more additional conditions expressed in the language of residuated structures. Also conversely, every left residuated l-groupoid satisfying the mentioned conditions can be organized into an orthomodular lattice.
\end{abstract}

2010 Mathematics Subject Classification: 06C15; 06A11

Keywords: orthomodular lattice, left residuated l-groupoid, divisibility, antitony, double negation law

It is well-known that residuated structures form an algebraic axiomatization of fuzzy logics, see e. g. [1] for an overview. The reader can find necessary concepts and definitions concerning residuated structures in [5], however this paper is selfcontained. Orthomodular lattices were introduced by G. Birkhoff and J. von Neumann as an algebraic axiomatization of the logic of quantum mechanics, see e. g. [4], [6] or [2] for details. Hence it is a natural question if these two concepts have a common base, i. e. if orthomodular lattices can be considered as residuated structures and hence as an axiomatization of certain fuzzy logic and, conversely, if certain residuated structures can be converted into orthomodular lattices, i. e. if the logic of quantum mechanics can be considered as a kind of fuzzy logic. For the theory of orthomodular lattices cf. the monographs [6] and [2] as well as the paper [3].

We start with the definition of an orthomodular lattice.

Definition 1. An orthomodular lattice is an algebra $\mathscr{L}=\left(L, \vee, \wedge,{ }^{\prime}, 0,1\right)$ of type $(2,2,1,0,0)$ satisfying (i) $-(\mathrm{v})$ for all $x, y \in L$ :

(i) $(L, \vee, \wedge, 0,1)$ is a bounded lattice.

(ii) $x \vee x^{\prime}=1$

(iii) $x \leq y$ implies $y^{\prime} \leq x^{\prime}$.

Support of the research of both authors by ÖAD, project CZ 04/2017, as well as by IGA, project PřF 2018 012, is gratefully acknowledged. Support of the research of the second author by the Austrian Science Fund (FWF), project I 1923-N25 entitled "New perspectives on residuated posets", is gratefully acknowledged. 
(iv) $\left(x^{\prime}\right)^{\prime}=x$

(v) $x \leq y$ implies $y=x \vee\left(y \wedge x^{\prime}\right)$.

Remark 1 . In every lattice $(L, \vee, \wedge)$ with a unary operation 'satisfying (iii) and (iv) the so-called de Morgan laws

$$
(x \vee y)^{\prime}=x^{\prime} \wedge y^{\prime} \text { and }(x \wedge y)^{\prime}=x^{\prime} \vee y^{\prime}
$$

hold.

Remark 2. According to the de Morgan laws condition (v) can be replaced by

(vi) $x \leq y$ implies $x=y \wedge\left(x \vee y^{\prime}\right)$.

Now we introduce left residuated l-groupoids.

Definition 2. A left residuated l-groupoid is an algebra $\mathcal{A}=(A, \vee, \wedge, \odot, \rightarrow, 0,1)$ of type $(2,2,2,2,0,0)$ satisfying (i) - (iii) for all $x, y, z \in A$ :

(i) $(A, \vee, \wedge, 0,1)$ is a bounded lattice.

(ii) $x \odot 1=1 \odot x=x$.

(iii) $x \odot y \leq z$ if and only if $x \leq y \rightarrow z$.

Condition (iii) is called left adjointness. $\mathcal{A}$ is said to satisfy divisibility if

$$
(x \rightarrow y) \odot x=x \wedge y
$$

for all $x, y \in A$. We define a unary operation ' on $A$ by

$$
x^{\prime}:=x \rightarrow 0
$$

for all $x \in A$. A is said to satisfy antitony if

$$
x \leq y \text { implies } y^{\prime} \leq x^{\prime}
$$

for all $x, y \in A$ and $\mathcal{A}$ is said to satisfy the double negation law if

$$
\left(x^{\prime}\right)^{\prime}=x
$$

for all $x \in A$.

Example 1. If $A:=\left\{0, a, a^{\prime}, b, b^{\prime}, 1\right\},(A, \vee, \wedge, 0,1)$ denotes the bounded lattice with the Hasse diagram

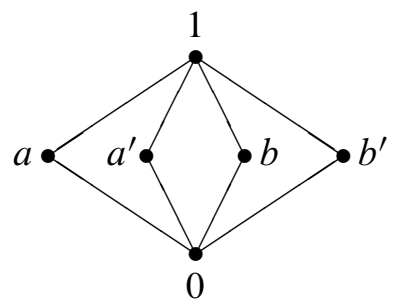


and the binary operations $\odot$ and $\rightarrow$ are defined by the tables

\begin{tabular}{c|cccccc}
$\odot$ & 0 & $a$ & $a^{\prime}$ & $b$ & $b^{\prime}$ & 1 \\
\hline 0 & 0 & 0 & 0 & 0 & 0 & 0 \\
$a$ & 0 & $a$ & 0 & $b$ & $b^{\prime}$ & $a$ \\
$a^{\prime}$ & 0 & 0 & $a^{\prime}$ & $b$ & $b^{\prime}$ & $a^{\prime}$ \\
$b$ & 0 & $a$ & $a^{\prime}$ & $b$ & 0 & $b$ \\
$b^{\prime}$ & 0 & $a$ & $a^{\prime}$ & 0 & $b^{\prime}$ & $b^{\prime}$ \\
1 & 0 & $a$ & $a^{\prime}$ & $b$ & $b^{\prime}$ & 1
\end{tabular}

\begin{tabular}{c|cccccc}
$\rightarrow$ & 0 & $a$ & $a^{\prime}$ & $b$ & $b^{\prime}$ & 1 \\
\hline 0 & 1 & 1 & 1 & 1 & 1 & 1 \\
$a$ & $a^{\prime}$ & 1 & $a^{\prime}$ & $a^{\prime}$ & $a^{\prime}$ & 1 \\
$a^{\prime}$ & $a$ & $a$ & 1 & $a$ & $a$ & 1 \\
$b$ & $b^{\prime}$ & $b^{\prime}$ & $b^{\prime}$ & 1 & $b^{\prime}$ & 1 \\
$b^{\prime}$ & $b$ & $b$ & $b$ & $b$ & 1 & 1 \\
1 & 0 & $a$ & $a^{\prime}$ & $b$ & $b^{\prime}$ & 1
\end{tabular}

then $(A, \vee, \wedge, \odot, \rightarrow, 0,1)$ is a left residuated l-groupoid satisfying divisibility, antitony and the double negation law. The mentioned lattice is the smallest orthomodular lattice which is not a Boolean algebra and it is usually denoted by MO2.

The following theorem says that to every orthomodular lattice there can be assigned a left residuated l-groupoid in a natural way.

Theorem 1. Let $\mathscr{L}=\left(L, \vee, \wedge,{ }^{\prime}, 0,1\right)$ be an orthomodular lattice and define binary operations $\odot$ and $\rightarrow$ on $L$ by the following formulas:

$$
\begin{aligned}
& x \odot y=\left(x \vee y^{\prime}\right) \wedge y, \\
& x \rightarrow y=(y \wedge x) \vee x^{\prime} .
\end{aligned}
$$

Then $\mathbf{A}(\mathscr{L})=(L, \vee, \wedge, \odot, \rightarrow, 0,1)$ is a left residuated l-groupoid satisfying divisibility, antitony, the double negation law as well as the following identity:

$$
x \odot(x \vee y)=x .
$$

Moreover, $x^{\prime}=x \rightarrow 0$ for all $x \in L$.

Proof. Let $a, b \in L$. We have

$$
a \rightarrow 0=(0 \wedge a) \vee a^{\prime}=0 \vee a^{\prime}=a^{\prime} .
$$

Of course, $(L, \vee, \wedge, 0,1)$ is a bounded lattice. Moreover,

$$
\begin{aligned}
& a \odot 1=\left(a \vee 1^{\prime}\right) \wedge 1=(a \vee 0) \wedge 1=a \wedge 1=a \text { and } \\
& 1 \odot a=\left(1 \vee a^{\prime}\right) \wedge a=1 \wedge a=a .
\end{aligned}
$$

If $a \odot b \leq c$ then $\left(a \vee b^{\prime}\right) \wedge b \leq c$ and hence

$$
a \leq a \vee b^{\prime}=\left(\left(a \vee b^{\prime}\right) \wedge b\right) \vee b^{\prime}=\left(\left(\left(a \vee b^{\prime}\right) \wedge b\right) \wedge b\right) \vee b^{\prime} \leq(c \wedge b) \vee b^{\prime}=b \rightarrow c \text {. }
$$

If, conversely, $a \leq b \rightarrow c$ then $a \leq(c \wedge b) \vee b^{\prime}$ and hence

$$
a \odot b=\left(a \vee b^{\prime}\right) \wedge b \leq\left(\left((c \wedge b) \vee b^{\prime}\right) \vee b^{\prime}\right) \wedge b=\left((c \wedge b) \vee b^{\prime}\right) \wedge b=c \wedge b \leq c .
$$

Now, using orthomodularity (i. e. (v) of Definition 1), we have

$$
(a \rightarrow b) \odot a=\left(\left((b \wedge a) \vee a^{\prime}\right) \vee a^{\prime}\right) \wedge a=\left((b \wedge a) \vee a^{\prime}\right) \wedge a=a \wedge b .
$$


In view of Definition $1, a \leq b$ implies $b^{\prime} \leq a^{\prime}$ and we have $\left(a^{\prime}\right)^{\prime}=a$. Finally, by applying (0.1) and (vi) of Remark 2 we obtain

$$
a \odot(a \vee b)=\left(a \vee(a \vee b)^{\prime}\right) \wedge(a \vee b)=a .
$$

Remark 3. The operation $x \odot y:=\left(x \vee y^{\prime}\right) \wedge y$ is called the Sasaki projection of $x$ onto $y$ (cf. [6] and [2]).

Conversely, certain left residuated l-groupoids give rise to an orthomodular lattice.

Theorem 2. Let $\mathcal{A}=(A, \vee, \wedge, \odot, \rightarrow, 0,1)$ be a left residuated l-groupoid satisfying antitony, the double negation law as well as identities (0.1) and (0.3) of Theorem 1. Moreover, define $x^{\prime}:=x \rightarrow 0$ for all $x \in A$. Then $\mathbf{L}(\mathcal{A})=\left(A, \vee, \wedge,{ }^{\prime}, 0,1\right)$ is an orthomodular lattice.

Proof. Let $a, b \in A$. Clearly, $(A, \vee, \wedge, 0,1)$ is a bounded lattice and $a^{\prime}=a \rightarrow 0$. Using antitony we see that $a \leq b$ implies $b^{\prime} \leq a^{\prime}$. Moreover, we have $\left(a^{\prime}\right)^{\prime}=a$ according to the double negation law. Finally, if $a \leq b$ then, using (0.3) and (0.1), we have

$$
b=\left(b^{\prime}\right)^{\prime}=\left(b^{\prime} \odot\left(b^{\prime} \vee a^{\prime}\right)\right)^{\prime}=\left(b^{\prime} \odot a^{\prime}\right)^{\prime}=\left(\left(b^{\prime} \vee a\right) \wedge a^{\prime}\right)^{\prime}=a \vee\left(b \wedge a^{\prime}\right)
$$

and hence $a \vee a^{\prime}=a \vee\left(1 \wedge a^{\prime}\right)=1$.

Finally, we prove that the correspondence described in the last two theorems is one-to-one.

Theorem 3. We have $\mathbf{L}(\mathbf{A}(\mathscr{L}))=\mathscr{L}$ for every orthomodular lattice $\mathscr{L}$ and $\mathbf{A}(\mathbf{L}(\mathscr{A}))=$ A for every left residuated $\mathrm{l}$-groupoid satisfying antitony, the double negation law as well as identities $(0.1)-(0.3)$ of Theorem 1.

Proof. If $\mathscr{L}=\left(L, \vee, \wedge,{ }^{\prime}, 0,1\right)$ is an orthomodular lattice, $\mathbf{A}(\mathscr{L})=(L, \vee, \wedge, \odot, \rightarrow$ $, 0,1)$ and $\mathbf{L}(\mathbf{A}(\mathscr{L}))=\left(L, \vee, \wedge,{ }^{*}, 0,1\right)$ then

$$
x^{*}=x \rightarrow 0=(0 \wedge x) \vee x^{\prime}=0 \vee x^{\prime}=x^{\prime}
$$

for all $x \in L$, therefore we obtain $\mathbf{L}(\mathbf{A}(\mathscr{L}))=\mathscr{L}$. Conversely, if $\mathscr{A}=(A, \vee, \wedge, \odot$, $\rightarrow, 0,1)$ is a left residuated l-groupoid satisfying divisibility, antitony, the double negation law as well as identities $(0.1)-(0.3)$ of Theorem $1, \mathbf{L}(\mathcal{A})=\left(A, \vee, \wedge,{ }^{\prime}, 0,1\right)$ and $\mathbf{A}(\mathbf{L}(\mathcal{A}))=(A, \vee, \wedge, \circ, \Rightarrow, 0,1)$ then

$$
\begin{aligned}
x \circ y & =\left(x \vee y^{\prime}\right) \wedge y=x \odot y \text { and } \\
x \Rightarrow y & =(y \wedge x) \vee x^{\prime}=x \rightarrow y
\end{aligned}
$$

for all $x, y \in A$, therefore we obtain $\mathbf{A}(\mathbf{L}(\mathcal{A}))=\mathcal{A}$. 
Remark 4. We have shown that orthomodular lattices can be considered as special residuated lattices and hence the logic of quantum mechanics axiomatized by them has a common base with a certain fuzzy logic axiomatized just by means of residuated lattices as pointed out in [1]. This sheds a new light on the logic of quantum mechanics and yields new tools for its investigation.

\section{REFERENCES}

[1] R. Belohlávek, Fuzzy relational systems. Foundations and principles. $\quad$ New York, NY: Kluwer Academic Publishers, 2002.

[2] L. Beran, "Orthomodular lattices. Algebraic approach." Mathematics and Its Applications (East European Series), 18. Dordrecht - Boston - Lancaster: D. Reidel Publishing Company, a member of the Kluwer Academic Publishers Group; Prague: Academia, Publishing House of the Czechoslovak Academy of Sciences. XIX, 394 p. Dfl. 180.00; \$ 69.00 (1985)., 1985.

[3] I. Chajda, R. Halaš, and J. Kühr, "Many-valued quantum algebras." Algebra Univers., vol. 60, no. 1, pp. 63-90, 2009, doi: 10.1007/s00012-008-2086-9.

[4] A. Dvurečenskij and S. Pulmannová, New trends in quantum structures. Dordrecht: Kluwer Academic Publishers; Bratislava: Ister Science, 2000.

[5] N. Galatos, P. Jipsen, T. Kowalski, and H. Ono, Residuated lattices. An algebraic glimpse at substructural logics. Amsterdam: Elsevier, 2007.

[6] G. Kalmbach, "Orthomodular lattices." London Mathematical Society Monographs, 18. London etc.: Academic Press Inc. (London) Ltd. VIII, 390 p. \$ 65.00 (1983)., 1983.

Authors' addresses

Ivan Chajda

Palacký University Olomouc, Faculty of Science, Department of Algebra and Geometry, 17. listopadu 12, 77146 Olomouc, Czech Republic

E-mail address: ivan.chajda@upol.cz

\section{Helmut Länger}

TU Wien, Faculty of Mathematics and Geoinformation, Institute of Discrete Mathematics and Geometry, Wiedner Hauptstraße 8-10, 1040 Vienna, Austria, and Palacký University Olomouc, Faculty of Science, Department of Algebra and Geometry, 17. listopadu 12, 77146 Olomouc, Czech Republic

E-mail address: helmut. laengeretuwien.ac.at 